Article

\title{
The Myelin Sheath Maintains the Spatiotemporal Fidelity of Action Potentials by Eliminating the Effect of Quantum Tunneling of Potassium Ions through the Closed Channels of the Neuronal Membrane
}

\author{
Abdallah Barjas Qaswal (D) \\ School of Medicine, The University of Jordan, Amman 11942, Jordan; qaswalabdullah@gmail.com
}

Received: 22 November 2019; Accepted: 5 December 2019; Published: 6 December 2019

\begin{abstract}
The myelin sheath facilitates action potential conduction along the axons, however, the mechanism by which myelin maintains the spatiotemporal fidelity and limits the hyperexcitability among myelinated neurons requires further investigation. Therefore, in this study, the model of quantum tunneling of potassium ions through the closed channels is used to explore this function of myelin. According to the present calculations, when an unmyelinated neuron fires, there is a probability of $9.15 \times 10^{-4}$ that it will induce an action potential in other unmyelinated neurons, and this probability varies according to the type of channels involved, the channels density in the axonal membrane, and the surface area available for tunneling. The myelin sheath forms a thick barrier that covers the potassium channels and prevents ions from tunneling through them to induce action potential. Hence, it confines the action potentials spatiotemporally and limits the hyperexcitability. On the other hand, lack of myelin, as in unmyelinated neurons or demyelinating diseases, exposes potassium channels to tunneling by potassium ions and induces the action potential. This approach gives different perspectives to look at the interaction between neurons and explains how quantum physics might play a role in the actions occurring in the nervous system.
\end{abstract}

Keywords: myelin; quantum tunneling; quantum biology; potassium ions; potassium channel; demyelination; nervous system; action potential

\section{Introduction}

The myelin sheath is a fat-rich layer that surrounds the neurons. It acts as an insulator by decreasing the ion flow by about 5000 -fold and enhances the velocity of action potential propagation along the axons. Therefore, action potentials do not occur in the myelinated membrane but they occur in the unmyelinated nodes of Ranvier [1]. Additionally, it was found that myelination is not only beneficial for increasing the velocity of electrical impulses but it is crucial for spatiotemporally confining action potentials and limiting the hyperexcitability among myelinated neurons, as in cortical pyramidal neurons [2]. Further evidence of this is the association between the increased tendency for seizures, which is a form of hyperexcitability, and demyelinating diseases, such as multiple sclerosis. However, the exact mechanism by which the myelin sheath exhibits its ability in limiting the hyperexcitability and confining the action potentials spatiotemporally is not explained well by the conventional background information on the functions of myelin [2-6]. Therefore, in this study, a different approach has been adopted to explain this unique function. This approach depends on the principles of quantum physics, particularly quantum tunneling, to be applied on ions.

Quantum physics has been introduced into different fields of science, such as biology and neuroscience, in an attempt to explain and understand many actions and processes occurring in the biological systems [7-13]. An important aspect of this which has gained more attention in recent years 
is the quantum behavior of ions in the channels of the biological membrane. This aspect aims to reveal the mechanism of the channels' selectivity filter [14-17] and to investigate the role of the voltage-gated channels when they are closed by applying the quantum tunneling phenomenon on ions [18-20].

Therefore, the aim of this study is to explore the role of myelin in maintaining the spatiotemporal fidelity of myelinated neurons and to explain different physiological and pathological actions that occur either due to myelination or demyelination. Furthermore, this study aims to fill the mathematical defects in the model used in referred pain [19]. These aims are achieved by using the model of quantum tunneling of ions through the closed gate of the channels [18].

\section{Methods}

The model of quantum tunneling of ions through the closed gate has been established [18] and will be used to investigate the function of myelin.

The classical idea of channel conductance is that channels permeate ions when their hydrophobic gate is open. However, it has been postulated that ions can pass through the closed gates via quantum tunneling, and then the tunneling current and quantum channel conductance can be calculated. This idea helped to explain the phenomenon of referred pain and the mechanism of action for lithium [18-20].

The tunneling probability through the closed gate can be calculated by the following equation:

$$
T=e^{-\frac{\sqrt{8 m}}{\hbar} \int_{X 1}^{X 2} \sqrt{q E x-E_{K}} d x}
$$

where $T$ is the tunneling probability; $m$ is the mass of the ion; $\hbar$ is the reduced Planck constant; $q$ is the charge of the ions; $E$ is the electric field that is needed to prevent the ion from passing the gate, corresponding to the value of gating free energy, which represents the energy needed to open the gate of the channel; $x$ is the ion position through the hydrophobic gate; $E_{K}$ is the kinetic energy of the ion; and $X 1$ to $X 2$ is the forbidden region where the ion cannot pass through the gate.

The ( $q E x)$ expression in Equation (1) represents the energy barrier of the passing ion. The electric field $E$ can be calculated by the following equation:

$$
E=\frac{U}{q L}
$$

where $U$ is the absolute value of gating free energy; which is the energy needed to open the closed gate; $q$ is the ion charge; and $L$ is the length of the gate, which is $4.4 \times 10^{-11} \mathrm{~m}$ [18].

Also, the forbidden region ( $X 1$ to $X 2$ ) is where the energy of the barrier is equal to or higher than the kinetic energy of the ions, as in the following equation:

$$
q E x \geq E_{K}
$$

Then, single channel conductance $C_{\text {Qion }}$ due to quantum tunneling can be calculated using the following equation:

$$
C_{\text {Qion }}=\frac{e^{2} T}{4 \pi^{2} \hbar}
$$

Finally, the quantum membrane conductance of ions $C_{Q M}$ can be calculated by the following equation:

$$
C_{Q M}=C_{Q i o n} \times D
$$

where $D$ is the channels density in the neuronal membrane. 


\section{Results}

\subsection{Increase in Extracellular Potassium Concentration during Action Potential}

When action potentials occur, sodium ions enter the neuron and potassium ions exit the neuron. This will increase the extracellular potassium concentration slightly [1]. There are $1.37 \times 10^{6}$ potassium ions per $314 \mu \mathrm{m}^{2}\left(4.36 \times 10^{3}\right.$ ions $\left./ \mu \mathrm{m}^{2}\right)$ of neuronal membrane surface area, which will exit to the extracellular space during the action potential [19]. For a neuron for which length $L=100 \mu \mathrm{m}$ and radius $r=0.5 \mu \mathrm{m}$, the neuronal membrane surface area $A=314 \mu \mathrm{m}^{2}$ and intracellular volume $V_{i}=78.5 \mu \mathrm{m}^{3}$ (assuming that the neuron takes the shape of a cylinder). Additionally, the ratio between the extracellular and intracellular volume varies from 0.59 to 0.75 [21,22]. Therefore, the extracellular volume $V_{o}=52.6 \mu \mathrm{m}^{3}$ (assuming that the ratio is 0.67 ). As a result, the slight increase in extracellular potassium concentration $\left[K^{+}\right]_{A P}$ can be calculated using the following equation:

$$
\left[K^{+}\right]_{A P}=\frac{N}{N_{A} V_{o}}
$$

where $N$ is the number of potassium ions that exit to the extracellular fluid per surface area unit per action potential, $N_{A}$ is the Avogadro's number, and $V_{o}$ is the extracellular volume.

By substituting the values in Equation (6), $\left[K^{+}\right]_{A P}=4.3 \times 10^{-2} \mathrm{mEq} / \mathrm{L}$.

\subsection{The Tunneling Probability of Potassium Ions through the Closed Gate of the Channels.}

The potassium ions, which come from the increase in potassium concentration, have the chance to tunnel through the closed gate of the potassium channels of the neurons that are adjacent to the stimulated ones. Therefore, the neurons that carry the electrical signals of action potentials will affect the neighboring neurons via quantum tunneling of potassium ions every time the neurons fire.

The tunneling probability of potassium ions will be calculated using Equation (1). However, first the energy barrier and the kinetic energy of the potassium ions must be calculated. Potassium voltage-gated channels Kv1.1 and Kv1.2 are clustered in the axons of neurons [23,24]. For that reason, the free gating energy of Kv1.2 channels will be used here to calculate the tunneling probability. Therefore, these channels need $7.7 \mathrm{Kcal} / \mathrm{mole}=5.35 \times 10^{-20} \mathrm{~J}$ to open their gate [25]. As a consequence, as in Equation (2), the corresponding electric field $E=7.6 \times 10^{9} \mathrm{~V} / \mathrm{m}$.

On the other hand, when extracellular potassium ions reach the hydrophobic gate, they will obtain kinetic energy due to the voltage of the neuronal membrane of $(-90) \mathrm{mV}$, because the gate is located at the intracellular end [26]. Thus, by neglecting the average thermal energy of potassium ions at body temperature on their total energy [19], the kinetic energy $\left(E_{K}\right)$ of extracellular potassium ions can be calculated by the following equation:

$$
E_{K}=q V
$$

where $q$ is the potassium ion charge and $V$ is the membrane voltage.

As a result, the kinetic energy $E_{K}=1.44 \times 10^{-20} \mathrm{~J}$. In addition, the forbidden region will be from $X_{1}=1.18 \times 10^{-11} \mathrm{~m}$ to $X_{2}=4.4 \times 10^{-11} \mathrm{~m}$. Accordingly, by solving Equation (1), the tunneling probability $T=2.1 \times 10^{-13}$.

\subsection{The Effect of Tunneling of Potassium Ions on the Resting Membrane Potential}

To show the effect of quantum tunneling of potassium ions on the resting membrane potential, membrane conductance due to quantum tunneling (quantum conductance) must be calculated by using Equations (4) and (5). If the potassium channel density of 50 channels $/ \mu \mathrm{m}^{2}$ [27] is substituted into the equations, the quantum membrane conductance $C_{Q M K}=6.5 \times 10^{-6} \mathrm{mS} / \mathrm{cm}^{2}$.

This value of conductance is $10^{9}$ higher than the one obtained in another study [18] because the type of voltage channel is different and consequently the energy barrier is different, even though this value of conductance will not affect the resting membrane potential because it is still much 
lower than the potassium and sodium conductance values, which are $0.5 \mathrm{mS} / \mathrm{cm}^{2}$ and $0.005 \mathrm{mS} / \mathrm{cm}^{2}$, respectively [1].

The Goldman-Hodgkin-Katz equation will be used here to calculate the threshold value of conductance of the extracellular potassium ions, which results from the increase in their concentration during action potential. This must affect the resting membrane voltage and induce the action potential, as in the following equation [1]:

$$
V(\text { millivolts })=-61 \times \log \frac{C_{N a}[N a]_{i}+C_{K}[K]_{i}}{C_{N a}[N a]_{o}+C_{K}[K]_{o}+C_{Q M K}[K]_{A P}}
$$

where $V$ is the membrane voltage, $C$ is the ion conductance at resting state, [ $]_{i}$ is the intracellular ion concentration, [ ] $]_{0}$ is the extracellular ion concentration, $C_{Q M K}$ is the quantum membrane conductance of potassium, and $[K]_{A P}$ is the increase in extracellular potassium concentration during the action potential.

Assuming that the threshold value of the membrane potential to induce the action potential is $-65 \mathrm{mV}$ [1], and by substituting the values $140,4,14,142,4.3 \times 10^{-2}, 0.5$, and 0.005 for the following variables $[K]_{i},[K]_{o},[N a]_{i},[N a]_{o},[K]_{A P}, C_{K}$, and $C_{N a}$ respectively [1], the threshold value of $C_{\mathrm{QMK}}$ will be $77.22 \mathrm{mS} / \mathrm{cm}^{2}$. If it is assumed for the sake of simplicity that at least one channel from the 50 channels $/ \mu \mathrm{m}^{2}$ is needed to achieve the threshold of conductance, then according to the equations; the tunneling probability must be at least $1.25 \times 10^{-4}$, which also represents the fraction of potassium ions that can tunnel to induce the action potential.

It is clear that the threshold value of conductance is larger than the actual obtained value, hence based on these calculations, the increase in extracellular potassium concentration during action potential will not affect the membrane potential and will not affect the excitability of neurons or induce the action potential. However, when neurons fire, action potentials become more frequent, and thus there will be a repetitive increase in potassium concentration over time. Therefore, from these repetitive trials, there is a probability that these potassium ions can achieve sufficient fractions that can tunnel through the closed gates and affect the membrane potential. Such a probability is calculated by using the Bernoulli trials equation:

$$
P(Z)=\frac{N ! P^{Z}(1-P)^{N-Z}}{(N-Z) ! Z !}
$$

where $N$ is the total number of trials, $Z$ is the number of successful trials, $P$ is the probability of success, and $P(Z)$ is the probability of achieving a certain number of successful trials. For example, if $Z=0$, then the equation will be $P(0)=(1-P)^{N}$.

To calculate the probability of inducing an action potential, the following steps should be considered:

1. The tunneling probability is $2.1 \times 10^{-13}$, which represents the probability $(P)$ of the potassium ion succeeding in passing the closed gate, but a fraction of $1.25 \times 10^{-4}$ is needed to induce the action potential. As mentioned before, there are $4.36 \times 10^{3}$ ions $/ \mu \mathrm{m}^{2}$ in extracellular fluid per action potential, and there are 50 channels $/ \mu \mathrm{m}^{2}$ in unmyelinated neurons. As a consequence, $N=87$ potassium ions will be trying to tunnel per channel. So, if at least one of these 87 potassium ions succeeds in tunneling, the fraction will be $1 / 87=1.15 \times 10^{-2}$, which is sufficient to induce action, since it is higher than $1.25 \times 10^{-4}$. So, the probability that at least one of the 87 potassium ions can tunnel through the closed gate is calculated as the following:

$$
\begin{aligned}
& P_{1}=1-P(0) \\
& P_{1}=1-\left(1-2.1 \times 10^{-13}\right)^{87} \\
& P_{1}=1.83 \times 10^{-11}
\end{aligned}
$$

2. Additionally, for the sake of simplicity it is assumed that one channel is enough to induce the action potential. Therefore, the probability that at least one channel from the 50 channels $/ \mu \mathrm{m}^{2}$ will be tunneled by a sufficient fraction of potassium ions is calculated as the following: 


$$
\begin{aligned}
& P_{2}=1-P_{1}(0) \\
& P_{2}=1-\left(1-1.83 \times 10^{-11}\right)^{50} \\
& P_{2}=9.15 \times 10^{-10}
\end{aligned}
$$

3. Finally, the probability of inducing the action potential also depends on the surface area available for quantum tunneling; the lager the surface area of the neuronal membrane, the higher the probability of inducing an action potential. As in previous points, the probability of inducing an action potential per $\mu \mathrm{m}^{2}$ is $9.15 \times 10^{-10}$.Therefore, for a surface area of $1 \mathrm{~mm}^{2}$ there will be $10^{6}$ times of (1) $\mu \mathrm{m}^{2}$ available for tunneling. Thus, the probability that at least one of these areas will be induced by an action potential, which will be enough to stimulate the adjacent areas of the neuron and transmit the signal to the brain, is calculated as the following:

$$
\begin{aligned}
& P_{3}=1-P_{2}(0) \\
& P_{3}=1-\left(1-9.15 \times 10^{-10}\right)^{10^{6}} \\
& P_{3}=9.15 \times 10^{-4}
\end{aligned}
$$

Therefore, this means that when the action potential happens in an unmyelinated neuron, there will be a probability of $9.15 \times 10^{-4}$ that this neuron will induce an action potential in a neighboring neuron when there is $1 \mathrm{~mm}^{2}$ surface area of neuronal membrane available for potassium ions to tunnel. This final value of the probability may be different when reasonable assumptions are made, as in the following:

(1) Assuming that the 87 potassium ions can hit the channels for (n) number of trials, here the total number of potassium ions trying to tunnel through the closed channels would be $87 \times n$ and the probability of inducing the action potential would change. For example, if $n=4$, then the total number of 348 potassium ions will try to tunnel, and if at least one of them tunnels, the fraction will be significant enough to affect the membrane potential, because $1 / 348=2.9 \times 10^{-3}$ is larger than $1.25 \times 10^{-4}$. By repeating the same sequence in the results section, the probability of inducing the action potential will be $3.6 \times 10^{-3}$. Also, if there must be at least (n) number of potassium ions available to tunnel for significant depolarization, then the probability can be calculated as the following:

$$
P_{1}=1-(P(0)+P(1)+P(2)+\ldots+P(n-1))
$$

(2) If one channel is not enough to affect the membrane potential and there must be at least (n) number of channels to be tunneled by potassium ions, then the probability can be calculated as the following:

$$
P_{2}=1-\left(P_{1}(0)+P_{1}(1)+P_{1}(2)+\ldots+P_{1}(n-1)\right)
$$

Moreover, this probability of inducing action potential varies according to the type of channels involved in tunneling, the density of the channels, and the surface area of the neuronal membrane available for tunneling.

\section{Discussion}

There are many physiological and pathological actions that support the hypothesis of quantum tunneling of potassium ions. One of these physiological actions that occur in the nervous system is the low spatial fidelity of the unmyelinated neurons of the anterolateral tract, which transmit slow pain, thermal sensation, crude touch, itching and sexual sensation. This is manifested as poor localization of the sensation and is extended to distant sites away from the original or local site, such as in referred pain and referred itching [1,19]. This might be explained as form of hyperexcitability due to lack of myelin in these neurons giving the opportunity for potassium ions to tunnel through the exposed potassium channels and generate a depolarization effect on adjacent neurons, and consequently action potentials occur. Thus, the brain cannot exactly identify the precise location of the stimulus. Also, quantum tunneling might affect the neurons that share the same spinal segments, hence causing the referred sensation. 
On the other hand, voltage-gated potassium channels in myelinated neurons are covered by a thick myelin sheath [28-30] that blocks the effect of quantum tunneling of potassium ions. As a result, potassium ions, which result from each action potential, will not tunnel through the closed channels, and consequently no action potentials will be induced. Hence, the myelinated neurons of the dorsal column medial lemniscus tract, which transmit fine touch, vibration, and proprioception, are characterized by high spatial fidelity with sharp and well localized sensations and absence of referred sensation phenomenon [1,19].

Moreover, when demyelination happens to the neurons, the potassium channels become exposed [31-34], and hence they are susceptible to tunneling by potassium ions, causing the action potential to be generated in demyelinated neurons. Therefore, myelin loss in the brain due to demyelinating diseases will result in hyperexcitability, manifested as an increased risk of seizures and epilepsy [3-6]. Additionally, it was found that demyelinated neurons exhibited ectopic action potentials that are generated distally in the axons and propagate antidromically towards the axon initial segment (AIS) and somatodendritic domain [2]. This supports the hypothesis of quantum tunneling of potassium ions, because when they tunnel through the exposed channels they induce action potentials randomly along the demyelinated axons away from the AIS. This means that myelin confines the action potentials spatiotemporally to the AIS by eliminating the quantum tunneling effect of potassium ions by forming a thick barrier that covers the channels.

\section{Summary}

According to the obtained results, it was found that when unmyelinated neuron fires, there is a probability that this neuron could induce an action potential in certain areas along the membrane of adjacent neurons via potassium ions tunneling through the closed channels. Therefore, this process would be responsible for the hyperexcitability among the unmyelinated neurons, and the degree of hyperexcitability would depend on the probability of action potential induction.

Funding: This research received no external funding.

Conflicts of Interest: The author declares no conflict of interest.

\section{References}

1. Hall, J.E. Guyton and Hall Textbook of Medical Physiology E-Book; Elsevier: Amsterdam, The Netherlands, 2015.

2. Hamada, M.S.; Kole, M.H. Myelin loss and axonal ion channel adaptations associated with gray matter neuronal hyperexcitability. J. Neurosci. 2015, 35, 7272-7286. [CrossRef] [PubMed]

3. Kelley, B.J.; Rodriguez, M. Seizures in patients with multiple sclerosis. CNS Drugs 2009, 23, 805-815. [CrossRef] [PubMed]

4. Lapato, A.S.; Szu, J.I.; Hasselmann, J.P.; Khalaj, A.J.; Binder, D.K.; Tiwari-Woodruff, S.K. Chronic demyelination-induced seizures. Neuroscience 2017, 346, 409-422. [CrossRef] [PubMed]

5. Hoffmann, K.; Lindner, M.; Gröticke, I.; Stangel, M.; Löscher, W. Epileptic seizures and hippocampal damage after cuprizone-induced demyelination in C57BL/6 mice. Exp. Neurol. 2008, 210, 308-821. [CrossRef] [PubMed]

6. Lebrun, C. Epilepsy and multiple sclerosis. Exp. Neurol. 2006, 8, 55-58.

7. Ingber, L. Evolution of regenerative Ca-ion wave-packet in neuronal-firing fields: Quantum path-integral with serial shocks. IJIRIS 2017, 4, 14-21. [CrossRef]

8. Ingber, L. Quantum calcium-ion interactions with EEG. Sci 2019, 1, 7.

9. Lambert, N.; Chen, Y.N.; Cheng, Y.C.; Li, C.M.; Chen, G.Y.; Nori, F. Quantum biology. Nat. Phys. 2013, 9, 10-18. [CrossRef]

10. Ball, P. The dawn of quantum biology. Nature 2011, 474, 272. [CrossRef]

11. McFadden, J.; Al-Khalili, J. Life on the Edge: the Coming of Age of Quantum Biology; Broadway Books: Portland, OR, USA, 2016. 
12. Chang, Y.F. Extensive quantum biology, applications of nonlinear biology and nonlinear mechanism of memory. NeuroQuantology 2012, 10, 183-189. [CrossRef]

13. Hameroff, S.; Penrose, R. Consciousness in the universe: A review of the 'Orch OR'theory. Phys. Life Rev. 2014, 11, 39-78. [CrossRef] [PubMed]

14. Vaziri, A.; Plenio, M.B. Quantum coherence in ion channels: resonances, transport and verification. New J. Phys. 2010, 12, 085001. [CrossRef]

15. Salari, V.; Naeij, H.; Shafiee, A. Quantum interference and selectivity through biological ion channels. Sci. Rep. 2017, 7, 41625. [CrossRef] [PubMed]

16. De March, N.; Prado, S.D.; Brunnet, L.G. Coulomb interaction rules timescales in potassium ion channel tunneling. J. Phys. Condens. Matter 2018, 30, 255101. [CrossRef]

17. Ganim, Z.; Tokmakoff, A.; Vaziri, A. Vibrational excitons in ionophores: experimental probes for quantum coherence-assisted ion transport and selectivity in ion channels. New J. Phys. 2011, 13, 113030. [CrossRef]

18. Qaswal, A.B. Quantum Tunneling of Ions through the Closed Voltage-Gated Channels of the Biological Membrane: A Mathematical Model and Implications. Quantum Reports 2019, 1, 219-225. [CrossRef]

19. Qaswal, A.B. A Theoretical Study to Explain the Referred Pain Phenomenon and Its Characteristics via Quantum Tunneling of Potassium Ions through the Channels of Neuronal Membrane. NeuroQuantology 2019, 17, 43-52.

20. Qaswal, A.B. Lithium stabilizes the mood of bipolar patients by depolarizing the neuronal membrane via quantum tunneling through the sodium channels. Clin. Psychopharmacol. Neurosci. Available online: http://www.cpn.or.kr/journal/view.html?uid=986\&vmd=Full\& (accessed on 22 November 2019).

21. Gligoroska, J.P.; Todorovska, L.; Mancevska, S.; Karagjozova, I.; Petrovska, S. Bioelectrical impedance analysis in karate athletes: BIA parameters obtained with inbody720 regarding the age. Res. Phys. Educ. Sport Health 2016, 5, 117-121.

22. Taniguchi, M.; Yamada, Y.; Fukumoto, Y.; Sawano, S.; Minami, S.; Ikezoe, T.; Watanabe, Y.; Kimura, M.; Ichihashi, N. Increase in echo intensity and extracellular-to-intracellular water ratio is independently associated with muscle weakness in elderly women. Eur. J. Appl. Physiol. 2017, 117, 2001-2007.

23. Rhodes, K.J.; Keilbaugh, S.A.; Barrezueta, N.X.; Lopez, K.L.; Trimmer, J.S. Association and colocalization of $\mathrm{K}+$ channel alpha-and beta-subunit polypeptides in rat brain. J. Neurosci. 1995, 15, 5360-5371. [CrossRef]

24. Rhodes, K.J.; Strassle, B.W.; Monaghan, M.M.; Bekele-Arcuri, Z.; Matos, M.F.; Trimmer, J.S. Association and colocalization of the $\mathrm{Kv} \beta 1$ and $\mathrm{Kv} \beta 2 \beta$-subunits with Kv1 $\alpha$-subunits in mammalian brain $\mathrm{K}+$ channel complexes. J. Neurosci. 1997, 17, 8246-8258. [CrossRef] [PubMed]

25. Islas, L.D. Functional diversity of potassium channel voltage-sensing domains. Channels 2016, 10, $202-213$. [CrossRef] [PubMed]

26. Labro, A.J.; Snyders, D.J. Being flexible: The voltage-controllable activation gate of $\mathrm{Kv}$ channels. Front. Pharmacol. 2012, 3, 168. [CrossRef]

27. Caldwell, J.H. Action Potential Initiation and Conduction in Axons; Elsevier: Amsterdam, The Netherlands, 2009; pp. 23-29.

28. Rasband, M.N.; Trimmer, J.S. Developmental clustering of ion channels at and near the node of Ranvier. Dev. Biol. 2001, 236, 5-16. [CrossRef]

29. Poliak, S.; Salomon, D.; Elhanany, H.; Sabanay, H.; Kiernan, B.; Pevny, L.; Stewart, C.L.; Xu, X.; Chiu, S.Y.; Shrager, P.; et al. Juxtaparanodal clustering of Shaker-like $\mathrm{K}+$ channels in myelinated axons depends on Caspr2 and TAG-1. J. Cell Biol. 2003, 162, 1149-1160. [CrossRef]

30. Rasband, M.N.; Trimmer, J.S.; Schwarz, T.L.; Levinson, S.R.; Ellisman, M.H.; Schachner, M.; Shrager, P. Potassium channel distribution, clustering, and function in remyelinating rat axons. J. Neurosci. 1998, 18, 36-47. [CrossRef]

31. Waxman, S.G. Membranes, myelin, and the pathophysiology of multiple sclerosis. N. Engl. J. Med. 1982, 306, 1529-1533. [CrossRef]

32. Ouyang, H.; Sun, W.; Fu, Y.; Li, J.; Cheng, J.X.; Nauman, E.; Shi, R. Compression induces acute demyelination and potassium channel exposure in spinal cord. J. Neurotrauma 2010, 27, 1109-1120. [CrossRef] 
33. Jukkola, P.I.; Lovett-Racke, A.E.; Zamvil, S.S.; Gu, C. K+ channel alterations in the progression of experimental autoimmune encephalomyelitis. Neurobiol. Dis. 2012, 47, 280-293. [CrossRef]

34. Shi, R.; Sun, W. Potassium channel blockers as an effective treatment to restore impulse conduction in injured axons. Neurosci. Bull. 2011, 27, 36-44. [CrossRef] 